\title{
Application of the Flory-Mandelkern Equation to Unfractionated Poly(vinyl alcohol) Samples
}

\author{
Maurycy Kalfus \\ Department of Chemistry, Faculty of Mathematics and Natural Sciences, \\ The Pedagogical College of Stupsk, 76-200 Stupsk, Poland.
}

(Received January 6, 1978)

\begin{abstract}
An iterative solution of the system of equations resulting from the FloryMandelkern equation and from the $\pi$-theorem was developed. The iteration involves experimental data for two unfractionated samples only, i.e., 2 values of intrinsic viscosity and 2 sets of sedimentation constants with the corresponding weight fractions. The efficiency of the iteration was shown for 21 samples of commercial poly(vinyl alcohol) (PVA) of different origin. Their degrees of hydrolysis were in the $97.7-99.4 \%$ range. The viscosity, sedimentation, and pycnometric measurements were done at $90^{\circ} \mathrm{C}$ in solutions which had been heated for 4 days in order to destroy the PVA associates. From sedimentation data at standard conditions, sedimentation constants were evaluated by a procedure which accounts for the concentration distribution along the ultracentrifuge cell due to the molecular mass distribution in the PVA samples. This in turn was determined from the sets of sedimentation constants and from the solution of the system of equations.
\end{abstract}

KEY WORDS: Flory-Mandelkern Equation / $\pi$-Theorem / Iteration / Sedimentation / Viscosity / Molecular Mass Distribution / Unfractionated Poly(vinyl alcohol) /

\section{THEORETICAL BACKGROUND}

Previous papers ${ }^{1-3}$ have discussed the application of the Flory-Mandelkern equation to unfractionated polymer samples in $\theta$-solutions ${ }^{1}$ and the generalized method of calculation of weightaverage molecular masses, $M_{w}$, of unfractionated polymer samples from sedimentation and viscosity measurements in non-ideal solutions ${ }^{2,3}$ and from measurements of other properties according to the $\pi$-theorem. ${ }^{3}$ These papers did not give for non-ideal solutions, a method of evaluating the exponent $\gamma$ in the proposed form of the Flory-Mandelkern equation

$$
M_{w}=K_{8 \eta} \bar{s}^{1.5}[\bar{\eta}]^{0.5}
$$

with

$$
\begin{gathered}
K_{\varepsilon \eta} \equiv\left[\frac{N \eta_{0}}{\Phi^{1 / 3} P^{-1}(1-v \rho)}\right]^{1.5} \\
\bar{s}=\left[\sum_{i=1}^{n} w_{i} s_{i}{ }^{1 / \gamma}\right]^{\gamma}
\end{gathered}
$$

and

$$
[\bar{\eta}]=[\eta] \bar{s}^{(2-3 r) / r} / \sum_{i=1}^{n} w_{i} s_{i}^{(2-3 r) / r}
$$

Here $\rho$ and $\eta_{0}$ are the density and viscosity of the solvent, $v$ is the partial specific volume of the polymer in the solvent, $N$ and $\Phi^{1 / 3} P^{-1}$ are the Avogadro and Flory-Mandelkern constants, [ $\eta]$ is the intrinsic viscosity, and $w_{i}$ and $s_{i}$ are the weight fractions and sedimentation constants which result from measurements of $n$ points in the sedimentation diagrams.

For a given polymer-solvent system at a given temperature, the constant $K_{s}$ can be obtained experimentally. The constant $\gamma$ cannot be evaluated from eq $1-4$, however, and the relations published $^{2}$ for the constants

$$
K_{s \eta} K_{s}{ }^{1.5} K_{\eta}{ }^{0.5}=1
$$

and

$$
1.5 \gamma+0.5 a=1
$$

which employ the equations:

$$
\bar{s}=K_{s} M_{w}{ }^{r}
$$


and

$$
[\bar{\eta}]=K_{\eta} M_{w}{ }^{a}
$$

do not yield directly the value of $\gamma$.

The system of eq $1-8$ is comparatively easy to solve only in the case of $\theta$-solutions, where

$$
\gamma=a=0.5
$$

Details of the procedure involved in the evaluation of $K_{s}$ and $K_{\eta}$ from experimental viscosity and sedimentation data for a single unfractionated sample have been published. ${ }^{1}$

\section{A General Solution of the Equation System}

The excluding of $K_{s \eta}$ from the discussed equations as a known constant, along with the corresponding eq 2 , results in a system of 7 equations with 7 unknown quantities: $M_{w} . \bar{s},[\vec{\eta}], \gamma, a, K_{s}$, and $K_{\eta}$. A solution of such a system should exist, but due to the form of eq 3 and 4 an analytical solution is not possible. Thus, a solution of the system by an iterative procedure was developed. It is stated here for the example of an investigation of 21 commercial poly(vinyl alcohol) (PVA) samples, done for a wider survey of the dependence of the mechanical properties of PVA on the molecular mass distribution (MMD). ${ }^{4}$ Some experimental and computational procedures and some theoretical problems discussed below for PVA may be similar to those encountered in the cases of other polymers, especially the ones which associate easily and are soluble only at higher temperatures.

In the case of PVA, the solution of the equation system was very vital, since PVA grades differ in the degree of hydrolysis, $i . e$, in the residual content of acidic groups. Thus, each PVA grade corresponds to a copolymer of vinyl alcohol with its esters and therefore for each PVA grade different values of the constants $\gamma, a, K_{s}$, and $K_{\eta}$ are to be expected and have to be evaluated. Due to the formation of associates ${ }^{5-9}$ and fiber-like crystals, temperatures close to $100^{\circ} \mathrm{C}$, at which no PVA associates exist, ${ }^{5}$ had to be applied during the measurements. Higher temperatures were advantageous for the sedimentation measurements of aqueous PVA solutions, due to the high $\eta_{0}$ value in water at low temperatures and its rapid decrease with increasing temperature, thus increasing the efficiency of the ultracentrifuge as a mass-spectrometer. Therefore, all sedimentation, viscosity, and other measurements were done at $90^{\circ} \mathrm{C}$, for which the values of the above constants were not known. ${ }^{10-17}$

The general iterative solution of the system of eq $1-8$ was based on the system of 2 equations with the 2 unknown quantities $K_{8, j}$ and $\gamma_{j}$.

$$
\begin{aligned}
& \log (\bar{s})_{j, 1}=\log K_{8, j}+\gamma_{j} \log \left(M_{w}\right)_{j, 1} \\
& \log (\bar{s})_{j, 2}=\log K_{8, j}+\gamma_{j} \log \left(M_{w}\right)_{j, 2}
\end{aligned}
$$

Where the subscript $j$ denotes the $j$-th iteration step and the subscripts 1 and 2 denote the numbers of two PVA samples which differ to such an extent that the experimental errors are insignificant in comparison with the difference $[\eta]_{2}-[\eta]_{1}$ and with the difference in sedimentation constants.

The $(\bar{s})_{j, k}$ and $\left(M_{w}\right)_{j, k}$ values with $k=1,2$, were computed from the experimental $[\eta], s_{i}$, and $w_{i}$ values according to eq $1-4$, with

$$
\gamma_{j-1}=\frac{\log \left[(\bar{s})_{j-1,2} /(\bar{s})_{j-1,1}\right]}{\log \left[\left(M_{w}\right)_{j-1,2} /\left(M_{w}\right)_{j-1,1}\right]}
$$

resulting from eq 10 at the preceding iteration step. The iteration was started with $\gamma_{0}=0.5$ and stopped when

$$
\frac{\gamma_{j}-\gamma_{j-1}}{\gamma_{j}}<d
$$

The data discussed in this paper were computed at $d=0.001$.

After the end of the iteration, the $K_{s}=K_{8, j}$ value was computed from eq 10 and the last $\gamma_{j}$ value at $k=2$ and then the $K_{\eta}$ and a values were computed from eq 5 and 6 , respectively.

\section{Evaluation of the Flory-Mandelkern Constant}

The evaluation of the $\Phi^{1 / 3} P^{-1}$ constant became necessary because of the following two facts.

(1) The constant is not quite universal, since its value depends on the flexibility of the macromolecular chains and therefore may be slightly different in different polymers. According to its chemical structure, PVA would be supposed to have rather flexible macromolecules, but some stiffness due to $\mathrm{H}$-bonds ${ }^{7,8}$ or ketal bonds ${ }^{18}$ could also be expected.

(2) The experimentally determined value of the constant depends on the MMD of the samples used in the measurements: in the case of flexible macromolecules it changes from $2.5 \times 10^{6}$ for broad fractions to $2.78 \times 10^{6}$ for monodisperse fractions. ${ }^{19}$ 
This difference results in a not quite negligible error in the $M_{w}$ value of $\pm 1.9 \%$.

Thus, the value of the constant was computed by starting from eq 1 and 2 and from published data $^{10,11}$ for $M, s$, and $[\eta]$ which are stated in Table I.

Table I. The Flory-Mandelkern constant computed for PVA from published data $^{10,11}$

\begin{tabular}{ccccc}
\hline Sample & $M \times 10^{-3}$ & $s, S$ & $\begin{array}{c}{[\eta],} \\
100 \mathrm{~cm}^{3} / \mathrm{g}\end{array}$ & $\begin{array}{c}\Phi^{1 / 3} P^{-1} \\
\times 10^{-6}\end{array}$ \\
\hline 1 & 90 & 2.09 & 1.20 & 2.57 \\
2 & 74.1 & 2.21 & 1.22 & 3.08 \\
3 & 34.4 & 1.42 & 0.79 & 2.88 \\
4 & 13.5 & 1.08 & 0.42 & 3.31 \\
5 & 564 & 5.40 & 2.40 & 2.57 \\
6 & 491 & 5.10 & 2.14 & 2.56 \\
7 & 410 & 4.55 & 1.97 & 2.51 \\
8 & 380 & 4.49 & 1.81 & 2.53 \\
9 & 347 & 4.03 & 1.72 & 2.37 \\
10 & 300 & 3.85 & 1.59 & 2.43 \\
11 & 291 & 3.97 & 1.58 & 2.56 \\
12 & 280 & 3.76 & 1.54 & 2.46 \\
13 & 278 & 3.64 & 1.56 & 2.41 \\
14 & 188 & 2.96 & 1.20 & 2.32 \\
15 & 140 & 2.56 & 1.10 & 2.38 \\
16 & 112 & 2.38 & 0.84 & 2.34 \\
17 & 105 & 2.13 & 0.90 & 2.24 \\
18 & 102 & 2.20 & 0.85 & 2.32 \\
\hline
\end{tabular}

a (1) Sample 1, an unfractionated sample; samples $2-4$, fractions of the sample.

(2) Data for samples $1-4$ refer $^{11}$ to $1-v \rho=0.235$ at $25^{\circ} \mathrm{C}$.

(3) Samples $5-18$, fractions. The corresponding data refer $^{10}$ to $1-v \rho=0.248$ at $20^{\circ} \mathrm{C}$.

Comparison of the values of the constant resulting from the data ${ }^{11}$ for a PVA sample and for its three fractions shows only that the values are higher and not too reliable: the average value for the three fractions is $(3.09 \pm 0.22) \times 10^{6}$. Thus, the theoretical value for monodisperse fractions of flexible macromolecules, $2.78 \times 10^{6}$, is near the lower limit of the computed values. If we compare this data with others in Table I, it is noteworthy that they refer to low $M$ values. A much better agreement of the values of the constant is found for other fractions ${ }^{10}$ in the table, especially for $M>$ 188,000 ; the average values of the constant are $(2.43 \pm 0.14) \times 10^{6}$ and $(2.49 \pm 0.12) \times 10^{6}:$ these correspond to broad fractions of flexible macromolecules. Thus, PVA macromolecules have enough flexibility in terms of the Flory-Mandelkern constant and therefore the value corresponding to flexible macromolecules is appropriate for PVA. Since $w_{i}$ and $s_{i}$ in eq 3 and 4 correspond to ideally monodisperse fractions, the theoretical value of the constant, $2.78 \times 10^{6}$, was assumed in this work.

The differences in the values of the constant in Table I are partially due to the unspecified averaging of $M, s$, and $[\eta]$ during their measurements.

\section{EXPERIMENTAL}

\section{Preparation of the PVA Solutions}

Thermal treatment of the aqueous PVA solutions is necessary for the decomposition of gels ${ }^{8}$ and associates. $^{5-9}$ Constant values of the weight average sedimentation constants, $s_{w}$, calculated from eq 3 at $\gamma=1$, were assumed in the corresponding experiments as a criterion for the selection of the thermal treatment conditions. During the heating at $90^{\circ} \mathrm{C}$ of the $4 \mathrm{mg} / \mathrm{cm}^{3}$ solution of sample 5 (Table II) in sealed measurement flasks of $500 \mathrm{~cm}^{3}$, the amount of rapidly sedimenting fractions and the $s_{w}$ values decreased, whereas the area under the curves in the sedimentation diagrams increased, showing an increase in the concentration of the true PVA solution. After 4 days of heating, the amount of the rapidly sedimenting fractions became negligible and $s_{w}$ reached the value of 5.99, which remained practically unchanged after the fifth day of heating. An additional sedimentation run after 17 days of heating at $90^{\circ} \mathrm{C}$ yielded $s_{w}=5.66$, i.e., a value by $5.5 \%$ lower than after 4 or 5 days of heating. Therefore in all subsequent experiments the $4 \mathrm{mg} / \mathrm{cm}^{3}$ solutions of the PVA samples were heated at $90^{\circ} \mathrm{C}$ during 4 days. The PVA samples were of $97.7-99.4 \%$ hydrolysis degree.

\section{The Sedimentation Measurements}

In a special series of experiments the standard sedimentation conditions, ${ }^{20}$ at which the diffusion and pressure effects were negligible and the concentration effect was small, were established to be $90 \pm 0.05^{\circ} \mathrm{C}$, PVA concentration $4 \mathrm{mg} / \mathrm{cm}^{3}$, rotation velocity $44700 \mathrm{rpm}$, and sedimentation time $76 \mathrm{~min}$. The meniscus position corresponded to 
$1.70 \mathrm{~cm}^{3}$ of the solution in $30-\mathrm{mm}$ high cells in the Spinco E ultracentrifuge with high-temperature equipment and Schlieren optics.

The temperature of $90^{\circ} \mathrm{C}$ was selected as the highest possible for the viscosity measurements stated below and for the transfer of the PVA solutions from the measurement flasks to the preheated ultracentrifuge cells by means of preheated syringe.

The evaluation procedure, i.e., the transformation of the coordinates of an $i$-th point in the sedimentation diagram into $s_{i}$ and $w_{i}$, differed from the previously published one $e^{1,20}$ only in the extrapolation of the experimental sedimentation coefficients, $s_{c, i}$, to infinite dilution, i.e., to $c=0$, yielding the sedimentation constants, $s_{i}$, as stated in eq 3 and 4 . The concentration dependence of $s_{c, i}$ was approximated for experimental data for $c$ equal to 4 and $6 \mathrm{mg} / \mathrm{cm}^{6}$ in 6 samples and for c equal to $2,4,5,6,7$, and $8 \mathrm{mg} / \mathrm{cm}^{3}$ in 2 samples, according to the equations ${ }^{21}$ :

$$
\begin{aligned}
& 1 / s_{c, i}=1 / s_{i}+k G\left(s_{i}\right) \\
& 1 / s_{c, i}=\left(1 / s_{i}\right)(1+k c) \\
& 1 / s_{c, i}=1 / s_{i}+k c
\end{aligned}
$$

and

$$
1 / s_{c, i}=\left(1 / s_{i}\right)\left[1+k c G\left(s_{i}\right)\right]
$$

Here $k$ was a constant, independent of the concentration, $c$, and

$$
G\left(s_{i}\right)=\sum_{j} w_{j}
$$

where the summation extended over the components $j$ whose sedimentation constants did not exceed $s_{i}$.

The changes in polymer concentration were neglected in spite of being substantial: from the inital concentration, $c$, in the sedimentation plateau region to 0 at the meniscus. Equations 13 and 16 accounted for the hitherto neglected changes in polymer concentration along the ultracentrifuge cell due to the MMD in the sample. Therefore, the dependence established from the sedimentation of separate fractions, with $s_{c, i}$ corresponding to the peak of the $i$-th fraction, ${ }^{22}$ may substantially differ from the one found in an unfractionated sample.

For the above PVA samples the best fit of experimental data was found for eq 16 with $k=0.08$.
This was used for the computation of the $s_{i}$ values.

\section{The Viscosity Measurements}

The $[\eta]$ values were evaluated from viscosity measurements of the aqueous PVA solutions at $90 \pm 0.01^{\circ} \mathrm{C}$ in hermetically closed viscometers. These were designed on the basis of one ${ }^{23}$ of the Ubbelhode type described previously. All dilutions of the initial solution of $10 \mathrm{mg} / \mathrm{cm}^{3}$, to 8,6 , 4 , and $2.5 \mathrm{mg} / \mathrm{cm}^{3}$, were done in the viscometer.

\section{The Determination of $K_{8 \eta}$}

The value of $v$ involved in eq 2 was 0.805 ; this was obtained from pyconometric data for 2, 1 , and $0.5 \%$ aqueous solutions of samples 3,20 , and 21 (Table II) at $90 \pm 0.01^{\circ} \mathrm{C}$. The other values in eq 2 were $N=6.024 \times 10^{23}$, the values published for water ${ }^{24}$ at $90^{\circ} \mathrm{C}, \eta_{0}=0.3165 \mathrm{cP}$ and $\rho=0.9653$, and the discussed $\Phi^{1 / 3} P^{-1}=2.78 \times 10^{6}$. The resulting $K_{8 \eta}=5393$ included the coefficient for expressing the sedimentation constants in Svedbergs, $S$, instead of seconds $\left(1 S=10^{-13} \mathrm{sec}\right)$.

\section{RESULTS AND DISCUSSION}

In order to select the most suitable samples for the iteration, the $(\bar{s})_{0}$ values were computed from eq $3 \gamma_{0}=0.5$ for all the 21 samples studied. The $(\bar{s})_{0}$ and $[\eta]$ values are stated in Table II. Samples 8 and 16 were selected as corresponding to the highest and lowest $(\bar{s})_{0}$ values at sufficiently reliable $[\eta]$ values (the $[\eta]$ value in sample 14 was too low). Thus in eq 11 and $12 k=1$ and $k=2$ correspond to samples 16 and 8 , respectively. The sedimentation constants and intrinsic viscosities are stated in Svedbergs and $100 \mathrm{~cm}^{3} / \mathrm{g}$, respectively.

The iteration yielded $\gamma_{1}=0.486, \gamma_{3}=0.443$, and $\gamma_{4}=0.443$ at the corresponding iteration steps. Thus, even the third iteration step yielded a $\gamma$ value satisfying the condition in eq 12 . With $\gamma=0.443$ and eq 10, 5, and 6 the following equations were obtained.

$$
\begin{aligned}
& \bar{s}=0.05098 M^{0.443} \\
& {[\bar{\eta}]=2.597 \times 10^{-4} M^{0.671}}
\end{aligned}
$$

With $\bar{s}$ and $M_{w}$ replaced by $s_{i}$ and $M_{i}$, eq 18 is applicable to the transformation of the sedimentation constants distribution (SCD) used in the iteration into MMD of the samples. The reliabi- 
Table II. Experimental and iteration viscosity and sedimentation data for commercial PVA samples

\begin{tabular}{|c|c|c|c|c|c|c|c|c|c|c|c|c|}
\hline \multirow{2}{*}{ Sample } & \multirow[b]{2}{*}{$s_{w}$} & \multirow[b]{2}{*}[\eta]{} & \multicolumn{3}{|c|}{$\gamma=0.5$} & \multicolumn{5}{|c|}{$\gamma=0.443$} & \multicolumn{2}{|c|}{ eq 20 and 21} \\
\hline & & & $(\bar{s})_{0}$ & {$[\eta]_{0}$} & $\begin{array}{l}\left(M_{w}\right)_{0} \\
\quad \times 10^{-3}\end{array}$ & $\bar{s}$ & {$[\bar{\eta}]$} & $M_{w}$ & $\log \bar{s}$ & $-\log [\bar{\eta}]$ & $\log \bar{s}^{\prime}$ & $-\log [\eta]^{\prime}$ \\
\hline 0 & 1 & 2 & 3 & 4 & 5 & 6 & 7 & 8 & 9 & 10 & 11 & 12 \\
\hline 1 & 4.27 & 0.24 & 4.97 & 0.279 & 31.6 & 5.18 & 0.276 & 33.4 & 0.7143 & 0.5591 & 0.7238 & 0.5874 \\
\hline 2 & 7.79 & 0.53 & 8.49 & 0.577 & 101.5 & 7.86 & 0.574 & 90.1 & 0.8954 & 0.2411 & 0.8959 & 0.2455 \\
\hline 3 & 8.25 & 0.65 & 8.85 & 0.699 & 118.7 & 8.10 & 0.697 & 104.0 & 0.9085 & 0.1568 & 0.9208 & 0.1961 \\
\hline 4 & 8.23 & 0.77 & 8.59 & 0.835 & 124.0 & 7.91 & 0.831 & 109.4 & 0.8982 & 0.1018 & 0.9296 & 0.1787 \\
\hline 6 & 6.77 & 0.35 & 7.77 & 0.402 & 74.0 & 7.33 & 0.394 & 67.2 & 0.8651 & 0.4045 & 0.8451 & 0.3465 \\
\hline 7 & 6.62 & 0.43 & 7.29 & 0.473 & 73.0 & 6.86 & 0.471 & 66.5 & 0.8363 & 0.3270 & 0.8432 & 0.3501 \\
\hline 8 & 8.51 & 0.54 & 9.19 & 0.583 & 114.8 & 8.32 & 0.581 & 98.8 & 0.9201 & 0.2358 & 0.9119 & 0.2137 \\
\hline ) & 7.51 & 0.43 & 8.10 & 0.464 & 84.7 & 7.60 & 0.463 & 76.9 & 0.8808 & 0.3344 & 0.8684 & 0.3001 \\
\hline 1) & 7.08 & 0.42 & 7.75 & 0.460 & 78.9 & 7.34 & 0.457 & 72.5 & 0.8657 & 0.3391 & 0.8582 & 0.3204 \\
\hline 11 & 6.66 & 0.35 & 7.20 & 0.378 & 64.1 & 6.94 & 0.378 & 60.6 & 0.8414 & 0.4225 & 0.8271 & 0.3821 \\
\hline 12 & 7.88 & 0.51 & 8.40 & 0.544 & 96.7 & 7.81 & 0.544 & 86.8 & 0.8927 & 0.2644 & 0.8894 & 0.2584 \\
\hline 13 & 4.22 & 0.20 & 4.89 & 0.232 & 28.1 & 5.12 & 0.230 & 29.9 & 0.7093 & 0.6383 & 0.7046 & 0.6254 \\
\hline 15 & 4.43 & 0.215 & 5.14 & 0.249 & 31.4 & 5.33 & 0.247 & 33.0 & 0.7267 & 0.6073 & 0.7217 & 0.7270 \\
\hline 16 & 4.24 & 0.249 & 4.89 & 0.287 & 31.3 & 5.14 & 0.284 & 33.3 & 0.7110 & 0.5467 & 0.7232 & 0.5884 \\
\hline 17 & 5.63 & 0.348 & 6.31 & 0.390 & 53.4 & 6.27 & 0.387 & 52.7 & 0.7980 & 0.4123 & 0.8029 & 0.4302 \\
\hline 18 & 7.13 & 0.472 & 7.92 & 0.524 & 87.0 & 7.45 & 0.519 & 79.0 & 0.8722 & 0.2848 & 0.8731 & 0.2908 \\
\hline 19 & 8.05 & 0.402 & 8.94 & 0.447 & 96.3 & 8.15 & 0.441 & 83.3 & 0.9112 & 0.3556 & 0.8823 & 0.2726 \\
\hline 20 & 6.51 & 0.432 & 7.24 & 0.480 & 72.9 & 6.85 & 0.477 & 66.8 & 0.8357 & 0.3206 & 0.8440 & 0.3486 \\
\hline 21 & 5.89 & 0.400 & 6.52 & 0.443 & 59.9 & 6.44 & 0.441 & 58.5 & 0.8089 & 0.3556 & 0.8210 & 0.3942 \\
\hline
\end{tabular}




\section{KaLfus}

lity of eq 18 must be checked in the cases of the transformation of SCD into MMD of other, even similar, PVA grades, since it was obtained from experimental data for 2 samples only. Therefore, eq 18 may not necessarily hold for other PVA grades, due to the effect of the differences in the hydrolysis degree and to other effects discussed in the theoretical background. A similar problem arises for grades of any copolymers which differ in composition. More reliable equations for different PVA grades of the $97.7-99.4 \%$ hydrolysis degree studied would be expected to result from experimental data for a larger number of different samples. The problem was investigated starting with experimental data for samples of $21 \mathrm{com}$ mercial PVA grades of different origin in the hydrolysis degree ranges studied. The experimental [ $\eta$ ] data, stated in column 2 of Table II, and the experimental $s_{i}$ data for the 21 samples were used in the investigation by computing the averaged $\bar{s}$ and $[\bar{\eta}]$ data according to eq 3 and 4 with the above $\gamma_{3}=\gamma_{4}=0.443$. From the resulting $\bar{s}$ and [ī] data, columns 6 and 7 , the $M_{w}$ data, column 8 , were computed for each sample according to eq 1 using the already determined $K_{s \eta}=5393$. Substantial differences in the hydrodynamic properties of the macromolecules in the PVA samples, due to their different hydrolysis degree and to other structural differences, would result in substantial differences in the relations stated in eq 7 and 8 , and therefore the graphs of $\log \bar{s}$ vs. $M_{w}$ and of $\log [\bar{\eta}]$ vs. $M_{w}$ for the 21 samples, or the corresponding treatment of the data by the least squares method, would yield values of the constants in eq 7 and 8 substantially different from those in eq 18 and 19, which were derived for a single pair of PVA samples. Computation of the above $M_{w}$, $\log \bar{s}$, and $\log [\bar{\eta}]$ data by the least squares method resulted in these new equations:

$$
\bar{s}=0.0826 M_{w}^{0.3994} \quad r=0.933
$$

and

$$
[\bar{\eta}]=6.682 \times 10^{-5} M_{w}{ }^{0.7931} \quad r=0.927
$$

By the same method the relation between the values in columns 2 and 8 was found.

$$
[\eta]=1.939 \times 10^{-5} M_{w}^{0.8996} \quad r=0.971
$$

In eq $20-22 r$ is the correlation coefficient: its high values show that the relations $20-22$ are very close to functional in spite of the different hydrolysis degree $(97.7-99.4 \%$ ) of the PVA samples, differences in their other features, e.g., in stereospecifity, and experimental errors in the viscosity and sedimentation data. The highest $r$ value in eq 22 shows the high reliability of the $[\eta]$ values, but since their averaging is unspecified, eq 22 may not necessarily yield $M_{w}$ for PVA samples of MMD other than those samples stated in Table II. The viscosity-average molecular masses, $M_{v}$, resulting from eq 22 will be close to $M_{w}$, however, since $a=0.897$ is close to $a=1$.

The values of $M_{w}, \log \bar{s}$, and $\log [\bar{\eta}]$, from which eq 20 and 21 were derived by the least squares method, are stated in columns 8,9 , and 10 . Substitution of the values in column 8 for $M_{w}$ in eq 20 and 21 yielded new $\log \bar{s}$ and $\log [\bar{\eta}]^{\prime}$ stated in columns 11 and 12 . They correspond to averaging according to eq 3 and 4 , respectively, at $\gamma=$ 0.3994 , whereas the preceding $\bar{s}$ and $[\bar{\eta}]$ values correspond to averaging at $\gamma=0.443$. The $\log \bar{s}$ and $\log \bar{s}^{\prime}$, as well as $\log [\bar{\eta}]$ and $\log [\bar{\eta}]^{\prime}$, differ markedly. With the values in columns 11 and 12 and with the resulting from them new $M_{w}$ values, calculated according to eq 1 , the least squares method yielded:

$$
\bar{s}^{\prime}=0.07754 M_{w}^{0.405} \quad r=0.969
$$

and

$$
[\bar{\eta}]^{\prime}=4.972 \times 10^{-5} M_{w}{ }^{0.8186} \quad r=0.916
$$

The $\gamma$ and $a$ values in eq 23 and 24 were within experimental errors ranges identical with those in eq 20 and 21, respectively, and therefore the repetition of the least squares procedure was useless. The mean values of the 21 sets of data in Table II yielded the following ratios: $[\bar{\eta}] /[\eta]]=1.103$, $\bar{s} /(\bar{s})_{0}=09578,[\bar{\eta}]^{\prime} /[\eta]=1.086,[\bar{\eta}]^{\prime} /[\bar{\eta}]=1.015$, and $\bar{s}^{\prime} / \bar{s}=0.9999, i . e$. , the values in the numerators and denominators of the fractions differed by 10.3, $4.2,8.6,1.5$, and $0.1 \%$, respectively. The relatively large first 3 values in both series show the necessity of the appropriate averaging, $i . e$., of the calculation of $\bar{s}$ and $[\bar{\eta}]$ according to eq 3 and 4 , respectively, whereas the much lower final 2 values in each series are within the experimental error ranges and show that the developed iterative procedure is sufficiently efficient and accurate and that the evaluation of the constants $\gamma, a, K_{8}$, and $K_{\eta}$ by the least squares method is superfluous. It 
is interesting to note here that the preexponential constants and exponents from eq 20 and 21 inserted in eq 5 and 6 yield 1.0462 and 0.9957 , respectively, in both cases instead of 1 (i.e., they agree with the $\pi$-theorem) whereas in the case of eq 23 and 24 the corresponding values are 0.8208 and 1.017 (i.e., only the exponents agree satisfactorily with the $\pi$-theorem).

Of course, this conclusion refers to aqueous PVA solutions at $90^{\circ} \mathrm{C}$, for which all the $\gamma$ values are not too far from 0.5 , corresponding to $\theta$-solution. The conclusion may be expanded practically to all sedimentation measurements, however, since at too small $\gamma$ values, corresponding to too good solvents, experimental errors in $w_{i}$ and $s_{i}$ values result in too great errors in $M_{w}$ values according to eq 3, 4, and 1. This is one of the reasons for limiting the application of an ultracentrifuge as a mass-spectrometer to $\theta$-solutions or to the vicinity of the $\theta$-region. ${ }^{20}$ The discussed $\gamma$ data show that the solution is not too far from the $\theta$-region for the determination of MMD of the PVA grades studied.

\section{CONCLUSION}

The developed iterative procedure, involving experimental data for two unfractionated samples of a polymer, is accurate and very efficient in solving the system of equations which result from the Flory-Mandelkern equation and from the $\pi$-theorem, and in yielding the molecular mass distributions of the polymer samples.

\section{REFERENCES}

1. M. Kalfus and J. Mitus, J. Polym. Sci. Part, $A-1$, 4, 953 (1966).

2. M. Kalfus and H. G. Elias, J. Macromol. Sci., Chem., A1, (No. 5), 955 (1967).

3. H. G. Elias and M. Kalfus, Makromol. Chem.,
105, 95 (1967).

4. D. W. Levi. "A Study of the Relation between Molecular Weight Distribution and Mechanical Properties of Poly (vinyl alcohol)," Doctor Thesis, Virginia Polytechnic Institute, U.S.A., 1954.

5. S. Kurosaki and T. Kawase, Kobunshi Kagaku, 13, 425 (1956).

6. E. B. Dyakonova, I. S. Okhrimenko, and I. E. Efremov, Vysokomol. Soedin., 7, 1016 (1965).

7. H. Buc, Annales de Chimie, 8, 431 (1963).

8. T. Matsuo and H. Inagaki, Makromol. Chem., 53, 131 (1962).

9. Y. Oyanagi and M. Matsumoto, Kobunshi Kagaku, 15, 348 (1958).

10. M. Schönberg, "Darstellung und Charakterisierung von Polyvinylalkoholfraktionen höheren Molekulargewichtes," Doctor Thesis, Technische Hochschule Hannover, FGR, 1955.

11. H. A. Dieu, J. Polym. Sci., 12, 417 (1954).

12. S. Kurosaki and K. Saiguchi, J. Chem. Soc. Jpn., Pure Chem. Sect., 75, 376 (1954).

13. K. Dialer, K. Vogler, and F. Patat, Helv. Chim. Acta, 35, 869 (1952).

14. T. Matsuo and H. Inagaki, Makromol. Chem., 55, 150 (1962).

15. G. Meyerhoff, Fortsch. der Hochpolym. Forschung, 3, 59 (1961).

16. A. Beresniewicz, J. Polym. Sci., 35, 321 (1959).

17. K. Staudinger and K. Warth, J. Prakt. Chem., 155, 261 (1940).

18. J. T. Clarke and E. R. Blout, J. Polym. Sci., 1, 419 (1946).

19. I. Ya. Poddubnyi and V. A. Grechanovskii, Vysokomol. Soedin., 6, 64 (1964).

20. M. Kalfus, Ann. Soc. Chim. Polonorum (in English), 39, 309 (1965).

21. I. Ya. Poddubnyi and V. A. Grechanovskii, Dokl. Akad. Nauk SSSR, 153, 1122 (1963).

22. J. W. Williams, K. E. van Holde, R. L. Baldwin, and H. Fujita, Chem. Rev., 58, 716 (1958).

23. J. Sachajdak and M. Kalfus, Przem. Chem., 41, 454 (1962).

24. E. Bingham and R. Jackson, Bull. Bureau Stand., 14, 75 (1918). 